\title{
Least Squares Weighted Residual Method for Solving the Generalised Elastic Column Buckling
} Problem

\author{
Charles C. Ike ${ }^{*}$, Clifford U. Nwoji ${ }^{2}$, Benjamin O. Mama², Hyginus N. Onah ${ }^{2}$ \\ ${ }^{1}$ Department of Civil Engineering, Faculty of Engineering, Enugu State University of Science and Technology, Enugu, 400001, \\ Enugu State, Nigeria \\ ${ }^{2}$ Department of Civil Engineering, University of Nigeria, Nsukka, Enugu State, Nigeria
}

Corresponding Author Email: ikecc2007@yahoo.com

https://doi.org/10.18280/ti-ijes.630111

Received: 19 January 2019

Accepted: 15 March 2019

\section{Keywords:}

Least squares weighted residual method, generalised elastic column buckling problem, asymmetric section, singly symmetric section, doubly symmetric section, characteristic buckling equation, algebraic eigenvalue eigenvector problem

\begin{abstract}
In this work, the least squares weighted residual method (LSWRM) was used to solve the generalised elastic column buckling problem for the case of pinned ends. Mathematically, the problem solved was a boundary value problem (BVP) represented by a system of three coupled linear ordinary differential equations (ODEs) in terms of three unknown displacement functions and subject to boundary conditions. The least squares residual method used formulated the problem as a variational problem, and reduced it to an algebraic eigenvalue problem which was solved to obtain the characteristic buckling equation. The characteristic stability equation was found to be a cubic polynomial for the general asymmetric sectioned column. The buckling modes were found as coupled flexural torsional buckling modes. Two special cases of the problem were studied namely: doubly symmetric and singly symmetric sections. For doubly symmetric sections, the buckling loads and the buckling mode were found to be decoupled and the buckling mode could be flexural or flexural - torsional. For singly symmetric section columns, one of the bucking modes becomes decoupled while the others are coupled. The buckling equation showed the column could fail by either pure flexure or coupled flexural - torsional buckling mode. The results of the present work agree with Timoshenko's results, and other results from the technical literature.
\end{abstract}

\section{INTRODUCTION}

\subsection{Background}

Thin walled structural members with open cross-sections which could be singly symmetric (mono-symmetric), bisymmetric (doubly symmetric) or asymmetric and which could be made of isotropic, anisotropic, homogeneous or nonhomogeneous materials are often encountered in engineering [1-5]. They could be used as beams, columns, or beam columns in buildings, machines, bridges, spacecraft, aerospace, aeronautics, naval and marine structures. Such structures are prone to failures by buckling under compression due to their thin-walled open cross-sections [6-7]. The general elastic thinwalled column buckling problem is governed by systems of complex, non-linear differential equations subject to boundary conditions. They demand formidable mathematical analysis and are difficult to solve. Closed form solutions don't exist for many of such problems.

Euler [8] was one of the pioneers in the research on the determination of the buckling loads and modal shapes of thin (slender) columns. He derived the equation of column buckling from equilibrium considerations, as an ordinary differential equation and solved it in closed form to obtain the buckling loads and buckling mode shapes. Saint Venant [9] studied the torsional behaviour of structures and presented buckling load and modal shape solutions for torsional buckling. Michell [10] and Prandtl [11] also formulated the mathematical problems describing the lateral stability of beams and derived solutions for flexural - torsional buckling for beams, columns and beam - columns. Timoshenko $[5,10-$ 12] presented the stability problem to incorporate warping torsion. The derivations and formulations of the mathematical equations to describe the flexural - torsional buckling of structures were accomplished by the research works due to Wagner [15], Timoshenko [5, 12-14], Vlasov [16], Timoshenko and Gere [5] Alsayed [17], Zlatko [18], Al Sheik [6], Trahair [19-20], Allen and Bulson [21] and Chajes [22]. Ike et al. [4] solved the generalised elastic column buckling problem using the Galerkin variational method for the case of column with pinned supports at the ends $x=0$, and $x=l$. They considered the case of an axial compressive load acting through the centroid, and such that the bimoment is zero and found that the Galerkin method simplified the problem to an algebraic eigenvalue eigenvector problem that was solved to obtain the characteristic buckling equation, with the roots as the buckling loads.

Mama et al. [3] used the finite Fourier sine transform method to solve the flexural - torsional buckling problem of thin-walled columns with pinned ends represented by the generalised elastic column buckling equations. They found that the finite Fourier sine transformation when applied to the system of governing linear ordinary differential equations transformed the problem to an algebraic eigenvalue eigenvector problem. They obtained non-trivial solutions to the homogeneous characteristic buckling equation in the form 
of a third order polynomial whose zeros are the buckling loads. Onah et al. [2] used the Fourier series method to solve the BVP of column buckling given by a system of governing linear ordinary differential equations. They solved the buckling problem of column with pinned ends, and found that the Fourier sine series method used transformed the Dirichlet boundary value problem to a system of algebraic homogeneous equations, representing an eigenvalue eigenvector problem. For nontrivial solutions, they obtained the characteristic buckling equation as a cubic polynomial in terms of the axial compressive load. They found the buckling loads as the three roots of the characteristic buckling equation.

Other researchers who have worked on buckling include: Mehmet [23], Nwakali [24], Howlett [25], Wang et al. [26], Det [27] and Zhu [28].

\subsection{Research aim and objectives}

The general aim of the study is to use the least squares weighted residual method to solve the generalised elastic column buckling problem, for an elastic column with pinned supports at the ends $x=0$, and $x=l$ where $l$ is the length of the column. The specific objectives are:

i. to formulate the boundary value problem (BVP) of elastic column buckling represented by a system of linear ordinary differential equations (ODE) in variational form using the least squares weighted residual method.

ii. to express the BVP as a least squares weighted residual problem using the least squares weighted residual variational statements for the BVP.

iii. to show that the least squares weighted residual variational statements simplify to an algebraic eigenvalue eigenvector problem, and solve the resulting algebraic eigenvalue problem to determine the characteristic buckling equation.

iv. to derive and solve expressions for the characteristic buckling equations for two cases, namely columns with doubly symmetric cross-sections and columns with singly symmetric cross-sections.

\section{THEORETICAL FRAMEWORK}

\subsection{Governing equations}

The generalised elastic column buckling problem formulated as a boundary value problem (BVP) in terms of the displaced configuration is represented mathematically by the following complex set of three coupled ordinary differential equations (ODEs) which are non-linear and obtained from an equilibrium analysis that disregards non-linear terms [1-5]:

$$
\begin{aligned}
& E\left(I_{y y} I_{z z}-I_{y z}^{2}\right) \frac{d^{4} v(x)}{d x^{4}}-I_{y y} N_{x} \frac{d^{2} v(x)}{d x^{2}}+I_{y z} N_{x} \frac{d^{2} w(x)}{d x^{2}}+ \\
& {\left[I_{y y} M_{l y}+I_{y z} M_{l z}-\right.} \\
& \left.N_{x}\left(e_{z} I_{y y}+e_{y} I_{y z}\right)\right] \frac{d^{2} \theta(x)}{d x^{2}}+\left(I_{y y} V_{z}+I_{y z} V_{y}\right) \frac{d \theta(x)}{d x}=q_{y} I_{y y}- \\
& q_{z} I_{y z}
\end{aligned}
$$

$$
\begin{aligned}
& E\left(I_{y y} I_{z z}-I_{y z}^{2}\right) \frac{d^{4} w(x)}{d x^{4}}-I_{z z} N_{y} \frac{d^{2} w(x)}{d x^{2}}+I_{y z} N_{x} \frac{d^{2} v(x)}{d x^{2}}+ \\
& {\left[-I_{z z} M_{l z}-I_{y z} M_{l z}+\right.} \\
& \left.N_{x}\left(e_{y} I_{z z}+e_{z} I_{y z}\right)\right] \frac{d^{2} \theta(x)}{d x^{2}}-\left(I_{z z} V_{y}-I_{y z} V_{z}\right) \frac{d \theta(x)}{d x}=q_{z} I_{z z}- \\
& q_{y} I_{y z}
\end{aligned}
$$

$$
\begin{aligned}
& \text { where } I_{E}=I_{y y}+I_{z z}+\left(e_{y}^{2}+e_{z}^{2}\right) A \\
& H_{y}=\iint_{A} z\left(y^{2}+z^{2}\right) d A \\
& H_{z}=\iint_{A} y\left(y^{2}+z^{2}\right) d A \\
& H_{w}=\iint_{A} 2\left(w_{0}-w\right)\left(y^{2}+z^{2}\right) d A \\
& C_{y}=\frac{I_{z z} H_{y}-I_{y z} H_{z}}{I_{y y} I_{z z}-I_{y z}^{2}}-2 e_{z} \\
& C_{z}=\frac{I_{y y} H_{z}-I_{y z} H_{y}}{I_{y y} I_{z z}-I_{y z}^{2}}-2 e_{y} \\
& I_{y y}=\iint_{A} z^{2} d A \\
& I_{z z}=\iint_{A} y^{2} d A \\
& I_{y z}=\iint_{A} y z d A
\end{aligned}
$$$$
E C_{w} \frac{d^{4} \theta(x)}{d x^{4}}-\left(G J+\frac{I_{E} N_{x}}{A}+C_{z} M_{l z}+C_{y} M_{l y}+\frac{H_{w} W_{w}}{C_{w}}\right) \frac{d^{2} \theta(x)}{d x^{2}}+
$$$$
\left(M_{l y}-e_{z} N_{x}\right) \frac{d^{2} v(x)}{d x^{2}}-\left(M_{l z}-e_{y} N_{x}\right) \frac{d^{2} w(x)}{d x^{2}}+V_{z} \frac{d v(x)}{d x}-
$$$$
V_{y} \frac{d w(x)}{d x}-\left(C_{z} V_{y}+C_{y} V_{z}+\frac{H_{w} V_{w}}{C_{w}}\right) \frac{d \theta(x)}{d x}=t_{x} \quad \ldots
$$

where $M_{l z}, M_{l y}$ are moments due to transverse loads only $J$ is the Saint Venant torsional stiffness of the cross-section $E$ is the Young's modulus of elasticity of the column material $G$ is the shear modulus or modulus of rigidity

$I_{y y}, I_{z z}$ are moments of inertia about the $y y$ and $z z$ axes

$C_{w}$ is the warping constant

$I_{E}$ is the polar moment of inertia about the shear centre

$I_{y z}$, is the product of inertia

$e_{y}, e_{z}$ are coordinates of the shear centre

$V_{y}, V_{z}$ are shear forces

$q_{y}, q_{z}$ are transverse loads

$N_{x}$ is the axial force

$v(x), w(x)$ are transverse displacement components in the $y$ and $z$ coordinate directions.

$\theta(x)$ is the twist or rotational displacement, $x$ represents the longitudinal axial coordinate variable

$y z$ is the plane of the cross-section of the column

$A$ is the area of the cross-section of the column

$t(x)$ is the applied torque.

The governing equations of equilibrium for the generalized elastic column buckling problem (non-prismatic cross-section and heterogeneous materials) is thus given by a system of three coupled simultaneous ordinary nonlinear differential equations with variable coefficients in terms of three unknown displacement functions $v(x), w(x)$ and $\theta(x)$. This system of governing equations is subject to the conditions imposed by the type of supports at the column ends. In column buckling problems involving columns with prismatic cross-sections and homogeneous materials, the elasticity properties $(G, J$ and $E$ ) as well as the inertial and geometrical properties become constants, but the load parameters $\left(M_{l y}, M_{l z}, V_{y}, V_{z}, W_{w}, V_{w}\right)$ are variables which are functions of axial longitudinal coordinate variable, $x$. The system of governing ordinary differential equations even for columns with prismatic cross-sections and homogeneous materials thus have variable coefficients, rendering them difficult to solve in closed-form.

Simplified forms of the system of governing ordinary 
differential equations of equilibrium are obtained by considering special characteristics of the different types of elastic column buckling problem. A simplified form of the system of governing equilibrium equations is obtained if the $y z$ coordinates of the cross-sectional plane are principal coordinates. Then, $I_{y z}=0$ and vanish from the governing equations of equilibrium yielding the simplified systems: [15].

$E I_{z z} \frac{d^{4} v(x)}{d x^{4}}-N_{x} \frac{d^{2} v(x)}{d x^{2}}+\left(M_{l y}-N_{x} e_{z}\right) \frac{d^{2} \theta(x)}{d x^{2}}+V_{z} \frac{d \theta(x)}{d x}=q_{y}$

$E I_{y y} \frac{d^{4} w(x)}{d x^{4}}-N_{x} \frac{d^{2} w(x)}{d x^{2}}+\left(N_{x} e_{y}-M_{l z}\right) \frac{d^{2} \theta(x)}{d x^{2}}-V_{y} \frac{d \theta(x)}{d x}=$ $q_{z}$

$$
\begin{aligned}
& E C_{w} \frac{d^{4} w(x)}{d x^{4}}-\left(G J+\frac{I_{E} N_{x}}{A}+C_{z} M_{l z}+C_{y} M_{l y}+\frac{H_{w} W_{w}}{C_{w}}\right) \frac{d^{2} \theta(x)}{d x^{2}}+\left(M_{l y}-e_{z} N_{x}\right) \frac{d^{2} v(x)}{d x^{2}}- \\
& \left(M_{l z}-e_{y} N_{x}\right) \frac{d^{2} w(x)}{d x^{2}}+V_{z} \frac{d v(x)}{d x}-V_{y} \frac{d w(x)}{d x}-\left(C_{z} V_{y}+C_{y} V_{z}+\frac{H_{w} V_{w}}{C_{w}}\right) \frac{d \theta(x)}{d x}=t(x)
\end{aligned}
$$

If the column is not subject to transverse loads, $q_{y}, q_{z}$ become zero; and the transverse moments are constants, then the shear forces $V_{y}, V_{z}$ also become zero. For columns subjected only to axially compressive force $N_{x}$ applied through the centroid of the cross-section, the moments due to the transverse loads would vanish and the applied torque also vanishes; and if the load is applied such that the bimoment is zero, the system of governing ordinary differential equations of equilibrium simplify further to become [1-5].

$E I_{z z} \frac{d^{4} v(x)}{d x^{4}}+N_{x} \frac{d^{2} v(x)}{d x^{2}}+N_{x} e_{z} \frac{d^{2} \theta(x)}{d x^{2}}=0$

$E I_{y y} \frac{d^{4} w(x)}{d x^{4}}+N_{x} \frac{d^{2} w(x)}{d x^{2}}-N_{x} e_{y} \frac{d^{2} \theta(x)}{d x^{2}}=0$

$E C_{w} \frac{d^{4} \theta(x)}{d x^{4}}-\left(G J-\frac{I_{E} N_{x}}{A}\right) \frac{d^{2} \theta(x)}{d x^{2}}+N_{x} e_{z} \frac{d^{2} v(x)}{d x^{2}}-$

$N_{x} e_{y} \frac{d^{2} w(x)}{d x^{2}}=0$

The system of governing ordinary differential equations of equilibrium given by Equations (16-18) represent a simplification of the generalized elastic column buckling problem presented by Equations (1-3) when $I_{y z}=0, q_{y}=$ $q_{z}=0, V_{y}=V_{z}=0, M_{l y}=M_{l z}=0, W_{w}=0, t(x)=0$

Let us define linear ordinary differential operators, $L_{1}$ and $L_{2}$ as:

$L_{1}=\frac{d^{4}}{d x^{4}}$

$L_{2}=\frac{d^{2}}{d x^{2}}$

Then the system of ordinary differential equations (Equations (16-18)) can be expressed in operator form as

$$
\begin{aligned}
& E I_{z z} L_{1} v(x)+N_{x} L_{2} v(x)+N_{x} e_{z} L_{1} \theta(x)=0 \\
& E I_{y y} L_{1} w(x)+N_{x} L_{2} w(x)-N_{x} e_{y} L_{2} \theta(x)=0
\end{aligned}
$$

$E C_{w} L_{1} \theta(x)-\left(G J-\frac{I_{E} N_{x}}{A}\right) L_{2} \theta(x)+N_{x} e_{z} L_{2} v(x)-$

$N_{x} e_{y} L_{2} w(x)=0$

where for a column of length, $l, 0 \leq x \leq l$ if one end of the column is considered as the origin of the longitudinal axial coordinate system. If the column material is homogeneous, the elastic properties $E$ and $G$ are constants, the system of governing equations. Equations (21-23) will be a linear system of ordinary differential equations with constant parameters.

\section{METHODOLOGY}

The least squares weighted residual methodology is based on seeking to obtain the minimum of the squares of the residual functions over the problem domain with respect to the unknown generalised displacement parameters. The three functionals to be minimized in this case become $F_{1}, F_{2}, F_{3}$ where

$$
\begin{aligned}
& F_{1}=\int_{0}^{l}\left(E I_{z z} L_{1} \bar{v}(x)+N_{x} L_{2} \bar{v}(x)+N_{x} e_{z} L_{1} \bar{\theta}(x)\right)^{2} d x \\
& F_{2}=\int_{0}^{l}\left(E I_{y y} L_{1} \bar{w}(x)+N_{x} L_{2} \bar{w}(x)-N_{x} e_{y} L_{2} \bar{\theta}(x)\right)^{2} d x \\
& F_{3}=\int_{0}^{l}\left(E C_{w} L_{1} \bar{\theta}(x)-\left(G J-\frac{I_{E} N_{x}}{A}\right) L_{2} \bar{\theta}(x)+\right. \\
& \left.N_{x} e_{z} L_{2} \bar{v}(x)-N_{x} e_{y} L_{2} w(x)\right)^{2} d x
\end{aligned}
$$

and $\bar{v}(x), \bar{w}(x)$ and $\bar{\theta}(x)$ are the approximate solutions to the three unknown functions.

If the approximations to the solutions are considered in the form

$\bar{v}(x)=\sum_{n=1}^{\infty} A_{n} \varphi_{n}(x)$

$\bar{w}(x)=\sum_{n=1}^{\infty} B_{n} \varphi_{n}(x)$

$\bar{\theta}(x)=\sum_{n=1}^{\infty} \theta_{n} \varphi_{n}(x)$

where $A_{n}, B_{n}$, and $\theta_{n}$ are the generalised displacement parameters for the appropriate displacement functions, $\bar{v}(x)$, $\bar{w}(x)$ and $\bar{\theta}(x)$ for the $n$th buckling mode, and $\varphi_{n}(x)$ are the buckling shape functions which are constructed to satisfy all the end boundary conditions (geometric and force boundary conditions), then, the least squares weighted residual variational equations become the system of three equations:

$\int_{0}^{l} \frac{\partial}{\partial A_{n}}\left(E I_{z z} L_{1} \bar{v}(x)+N_{x} L_{2} \bar{v}(x)+N_{x} e_{z} L_{1} \bar{\theta}(x)\right)^{2} d x=0$

$\int_{0}^{l} \frac{\partial}{\partial B_{n}}\left(E I_{y y} L_{1} \bar{w}(x)+N_{x} L_{2} \bar{w}(x)-N_{x} e_{y} L_{2} \bar{\theta}(x)\right)^{2} d x=0$

$\int_{0}^{l} \frac{\partial}{\partial \theta_{n}}\left(E C_{w} L_{1} \bar{\theta}(x)-\left(G J-\frac{I_{E} N_{x}}{A}\right) L_{2} \bar{\theta}(x)+N_{x} e_{z} L_{2} \bar{v}(x)-\right.$

$\left.N_{x} e_{y} L_{2} \bar{w}(x)\right)^{2} d x=0$ 
Simplifying, it is obtained that:

$\sum_{n=1}^{\infty} \int_{0}^{l}\left(E I_{z Z} L_{1} A_{n} \varphi_{n}(x)+N_{x} L_{2} A_{n} \varphi_{n}(x)+\right.$

$\left.N_{x} e_{z} L_{1} \theta_{n} \varphi_{n}(x)\right) \varphi_{n}(x) d x=0$

$\sum_{n=1}^{\infty} \int_{0}^{l}\left(E I_{y y} L_{1} B_{n} \varphi_{n}(x)+N_{x} L_{2} B_{n} \varphi_{n}(x)-\right.$

$\left.N_{x} e_{y} L_{2} \theta_{n} \varphi_{n}(x)\right) \varphi_{n}(x) d x=0$

$\sum_{n=1}^{\infty} \int_{0}^{l}\left(E C_{w} L_{1} \theta_{n} \varphi_{n}(x)-\left(G J-\frac{I_{E} N_{x}}{A}\right) L_{2} \theta_{n} \varphi_{n}(x)\right.$

$\left.N_{x} e_{y} L_{2} \theta_{n}(x)\right) \varphi_{n}(x) d x=0$

\section{RESULTS}

4.1 Application of the least squares weighted residual method to the general elastic column buckling problem with pinned supported ends at $x=0$ and $x=l$

For the generalised elastic column buckling problem where the ends $x=0$ and $x=l$ are on pinned supports the deflection (geometric) and force boundary conditions are:

$v(x=0)=0$

$w(x=0)=0$

$\theta(x=0)=0$

$v(x=l)=0$

$w(x=l)=0$

$\theta(x=l)=0$

$v^{\prime \prime}(x=0)=0$

$w^{\prime \prime}(x=0)=0$

$\theta^{\prime \prime}(x=l)=0$

$v^{\prime \prime}(x=l)=0$

$w^{\prime \prime}(x=l)=0$

$\theta^{\prime \prime}(x=l)=0$

Suitable displacement functions that satisfy these boundary conditions are taken as the infinite series:

$\bar{v}(x)=\sum_{n=1}^{\infty} A_{n} \sin \frac{n \pi x}{l}$

$\bar{w}(x)=\sum_{n=1}^{\infty} B_{n} \sin \frac{n \pi x}{l}$

$\bar{\theta}(x)=\sum_{n=1}^{\infty} \theta_{n} \sin \frac{n \pi x}{l}$

It is thus observed that the displacement shape functions that satisfy the boundary conditions of pinned supports at the ends $x=0$, and $x=l$ for the three displacement functions can be given for all possible buckling modes, $n$, by:

$\varphi_{n}(x)=\sin \frac{n \pi x}{l}$

where, $n=1,2,3,4,5,6,7,8,9, \ldots$

Application of the linear differential operators yield:

$L_{1} \varphi_{n}(x)=\frac{d^{4}}{d x^{4}} \sin \frac{n \pi x}{l}=\left(\frac{n \pi}{l}\right)^{4} \sin \frac{n \pi x}{l}$

$L_{1} \varphi_{n}(x)=\left(\frac{n \pi}{l}\right)^{4} \varphi_{n}(x)$

$L_{2} \varphi_{n}(x)=\frac{d^{2}}{d x^{2}} \sin \left(\frac{n \pi x}{l}\right)=-\left(\frac{n \pi}{l}\right)^{2} \sin \frac{n \pi x}{l}$

$L_{2} \varphi_{n}(x)=-\left(\frac{n \pi}{l}\right)^{2} \varphi_{n}(x)$

By substitution into Equations (33-35), the least squares weighted residual variational integrals become the system:

$\sum_{n=1}^{\infty} \int_{0}^{l}\left(E I_{z z} A_{n}\left(\frac{n \pi}{l}\right)^{4} \sin \frac{n \pi x}{l}-N_{x} A_{n}\left(\frac{n \pi}{l}\right)^{2} \sin \frac{n \pi x}{l}-\right.$

$\left.N_{x} e_{z} \theta_{n}\left(\frac{n \pi}{l}\right)^{2} \sin \frac{n \pi x}{l}\right) \times$

$\sin \frac{n \pi x}{l} d x=0$

$\sum_{n=1}^{\infty} \int_{0}^{l}\left(E I_{y y} B_{n}\left(\frac{n \pi}{l}\right)^{4} \sin \frac{n \pi x}{l}-N_{x} B_{n}\left(\frac{n \pi}{l}\right)^{2} \sin \frac{n \pi x}{l}+\right.$

$\left.N_{x} e_{y} \theta_{n}\left(\frac{n \pi}{l}\right)^{2} \sin \frac{n \pi x}{l}\right) \times$

$\sin \frac{n \pi x}{l} d x=0$

$\sum_{n=1}^{\infty} \int_{0}^{l}\left(E C_{w} \theta_{n}\left(\frac{n \pi}{l}\right)^{4} \sin \frac{n \pi x}{l}+(G J-\right.$

$\left.\frac{I_{E} N_{x}}{A}\right) \theta_{n}\left(\frac{n \pi}{l}\right)^{2} \sin \frac{n \pi x}{l}-N_{x} e_{z} A_{n}\left(\frac{n \pi}{l}\right)^{2} \sin \frac{n \pi x}{l}+$

$\left.N_{x} e_{y} B_{n}\left(\frac{n \pi}{l}\right)^{2} \sin \frac{n \pi x}{l}\right) \sin \frac{n \pi x}{l} d x=0$

Simplifying,

$\sum_{n=1}^{\infty}\left(E I_{z z} A_{n}\left(\frac{n \pi}{l}\right)^{4}-N_{x} A_{n}\left(\frac{n \pi}{l}\right)^{2}-\right.$

$\left.N_{x} e_{z} \theta_{n}\left(\frac{n \pi}{l}\right)^{2}\right) \int_{0}^{l} \sin ^{2} \frac{n \pi x}{l} d x=0$

$\sum_{n=1}^{\infty}\left(E I_{y y} B_{n}\left(\frac{n \pi}{l}\right)^{4}-N_{x} B_{n}\left(\frac{n \pi}{l}\right)^{2}+\right.$

$\left.N_{x} e_{y} \theta_{n}\left(\frac{n \pi}{l}\right)^{2}\right) \int_{0}^{l} \sin ^{2} \frac{n \pi x}{l} d x=0$

$\sum_{n=1}^{\infty}\left(E C_{w} \theta_{n}\left(\frac{n \pi}{l}\right)^{4}+\left(G J-\frac{I_{E} N_{\chi}}{A}\right) \theta_{n}\left(\frac{n \pi}{l}\right)^{2}-\right.$

$\left.N_{x} e_{z} A_{n}\left(\frac{n \pi}{l}\right)^{2}+N_{x} e_{y} B_{n}\left(\frac{n \pi}{l}\right)^{2}\right) \times$

$\int_{0}^{l} \sin ^{2} \frac{n \pi x}{l} d x=0$

Division by $\left(\frac{n \pi}{l}\right)^{2}$ yields: 
$\sum_{n=1}^{\infty}\left(\left(E I_{z z}\left(\frac{n \pi}{l}\right)^{2}-N_{x}\right) A_{n}-N_{x} e_{z} \theta_{n}\right) I_{n}=0$

$\sum_{n=1}^{\infty}\left(\left(E I_{y y}\left(\frac{n \pi}{l}\right)^{2}-N_{x}\right) B_{n}+N_{x} e_{y} \theta_{n}\right) I_{n}=0$

$\sum_{n=1}^{\infty}\left(\left(E C_{w}\left(\frac{n \pi}{l}\right)^{2}+\left(G J-\frac{I_{E} N_{x}}{A}\right)\right) \theta_{n}-N_{x} e_{z} A_{n}+\right.$

$\left.N_{x} e_{y} B_{n}\right) I_{n}=0$

where $I_{n}=\int_{0}^{l} \sin ^{2} \frac{n \pi x}{l} d x$

For any arbitrary buckling mode, the problem reduces to the following system of algebraic homogeneous equations in terms of the displacement modal amplitudes $A_{n}, B_{n}$ and $\theta_{n}$ :

$$
\begin{aligned}
& \left(E I_{z z}\left(\frac{n \pi}{l}\right)^{2}-N_{x}\right) A_{n}-N_{x} e_{z} \theta_{n}=0 \\
& \left(E I_{y y}\left(\frac{n \pi}{l}\right)^{2}-N_{x}\right) B_{n}+N_{x} e_{y} \theta_{n}=0 \\
& \left(E C_{w}\left(\frac{n \pi}{l}\right)^{2}+\left(G J-\frac{I_{E} N_{x}}{A}\right)\right) \theta_{n}-N_{x} e_{z} A_{n}+N_{x} e_{y} B_{n}=0
\end{aligned}
$$

In matrix form, we obtain the homogeneous equation:

$$
\left(\begin{array}{ccc}
\left(E I_{z z}\left(\frac{n \pi}{l}\right)^{2}-N_{x}\right) & 0 & -N_{x} e_{z} \\
0 & \left(E I_{y y}\left(\frac{n \pi}{l}\right)^{2}-N_{x}\right) & N_{x} e_{y} \\
-e_{z} N_{x} & e_{y} N_{x} & \left(E C_{w}\left(\frac{n \pi}{l}\right)^{2}+G J-\frac{I_{E} N_{x}}{A}\right)
\end{array}\right)\left(\begin{array}{l}
A_{n} \\
B_{n} \\
\theta_{n}
\end{array}\right)=\left(\begin{array}{l}
0 \\
0 \\
0
\end{array}\right)
$$

For non trivial solutions, the determinant of the coefficient matrix in Equation (69) is required to vanish yielding the stability equation as follows:

$$
\left|\begin{array}{ccc}
\left.E I_{z z}\left(\frac{n \pi}{l}\right)^{2}-N_{x}\right) & 0 & -N_{x} e_{z} \\
0 & \left(E I_{y y}\left(\frac{n \pi}{l}\right)^{2}-N_{x}\right) & N_{x} e_{y} \\
-N_{x} e_{z} & N_{x} e_{y} & \left(E C_{w}\left(\frac{n \pi}{l}\right)^{2}+G J-\frac{I_{E} N_{x}}{A}\right)
\end{array}\right|=0
$$

Let $Q_{z z}=E I_{z z}\left(\frac{n \pi}{l}\right)^{2}$

$Q_{y y}=E I_{y y}\left(\frac{n \pi}{l}\right)^{2}$

$$
Q_{\phi}=\frac{A}{I_{E}}\left(E C_{w}\left(\frac{n \pi}{l}\right)^{2}+G J\right)
$$

Then, $E C_{w}\left(\frac{n \pi}{l}\right)^{2}+G J=\frac{Q_{\phi} I_{E}}{A}$ where, $Q_{z z}$ is the Euler flexural buckling load about the $z z$ axis, $Q_{y y}$ is the Euler flexural buckling load about the $y y$ axis and $Q_{\phi}$ is the torsional buckling load.

The stability (buckling) equation then becomes:

$$
\left|\begin{array}{ccc}
\left(Q_{z z}-N_{x}\right) & 0 & -N_{x} e_{z} \\
0 & \left(Q_{y y}-N_{x}\right) & N_{x} e_{y} \\
-N_{x} e_{z} & N_{x} e_{y} & \left(\frac{Q_{\phi} I_{E}}{A}-\frac{N_{x} I_{E}}{A}\right)
\end{array}\right|=0
$$

Expansion of the stability equations gives:

$\left(Q_{z z}-N_{x}\right)\left|\begin{array}{cc}Q_{y y}-N_{x} & N_{x} e_{y} \\ N_{x} e_{y} & \frac{I_{E}}{A}\left(Q_{\phi}-N_{x}\right)\end{array}\right|-N_{x} e_{z}\left|\begin{array}{cc}0 & \left(Q_{y y}-N_{x}\right) \\ -N_{x} e_{z} & N_{x} e_{y}\end{array}\right|=0$

Further expansion yields:

$$
\begin{aligned}
& \left(Q_{z z}-N_{x}\right)\left[\left(Q_{y y}-N_{x}\right)\left(Q_{\phi}-N_{x}\right) \frac{I_{E}}{A}-\left(N_{x} e_{y}\right)^{2}\right]-N_{x} e_{z}\left(0+N_{x} e_{z}\left(Q_{y y}-N_{x}\right)\right)=0 \\
& \left(Q_{z z}-N_{x}\right)\left(Q_{y y}-N_{x}\right)\left(Q_{\phi}-N_{x}\right) \frac{I_{E}}{A}-\left(N_{x} e_{y}\right)^{2}\left(Q_{z z}-N_{x}\right)-\left(N_{x} e_{z}\right)^{2}\left(Q_{y y}-N_{x}\right)=0 \\
& \left(Q_{z z}-N_{x}\right)\left(Q_{y y}-N_{x}\right)\left(Q_{\phi}-N_{x}\right)-N_{x}^{2} e_{y}^{2} \frac{A}{I_{E}}\left(Q_{z z}-N_{x}\right)-N_{x}^{2} e_{z}^{2} \frac{A}{I_{E}}\left(Q_{y y}-N_{x}\right)=0
\end{aligned}
$$

But $r_{0}^{2}=e_{y}^{2}+e_{z}^{2}+\left(\frac{I_{x x}+I_{y y}}{A}\right)$

From Equation (4),

$$
\frac{I_{E}}{A}=\frac{I_{x x}+I_{y y}+\left(e_{x}^{2}+e_{y}^{2}\right) A}{A}=\frac{I_{x x}+I_{y y}}{A}+e_{x}^{2}+e_{y}^{2}=r_{0}^{2}
$$

Hence,

$$
\left(Q_{z z}-N_{x}\right)\left(Q_{y y}-N_{x}\right)\left(Q_{\phi}-N_{x}\right)-\frac{N_{x}^{2} e_{y}^{2}}{r_{0}^{2}}\left(Q_{z z}-N_{x}\right)-N_{x}^{2} \frac{e_{z}^{2}}{r_{0}^{2}}\left(Q_{y y}-N_{x}\right)=0
$$

Simplifying,

$\left(Q_{z z}-N_{x}\right)\left[\left(Q_{y y}-N_{x}\right)\left(Q_{\phi}-N_{x}\right)-N_{x}^{2} \frac{e_{y}^{2}}{r_{0}^{2}}\right]-N_{x}^{2} \frac{e_{z}^{2}}{r_{0}^{2}}\left(Q_{y y}-N_{x}\right)=0$

or, $\left(Q_{y y}-N_{x}\right)\left[\left(Q_{z z}-N_{x}\right)\left(Q_{\phi}-N_{x}\right)-N_{x}^{2} \frac{e_{z}^{2}}{r_{0}^{2}}\right]-$

$N_{x}^{2} \frac{e_{y}^{2}}{r_{0}^{2}}\left(Q_{z z}-N_{x}\right)=0$

Equation (82) is the characteristic buckling equation for finding the buckling loads of a generalized elastic column with an asymmetric cross-section and pinned supported ends at $x=0$ and $x=l$. The equation is a third order polynomial in $N_{x}$ with three roots. The three roots of the characteristic buckling equation are determined using the methods of solving polynomial equations. The smallest of the three buckling loads will govern the buckling behaviour of the column.

Two particular cases of the generalized elastic column buckling problem, which are simplifications of the general flexural - torsional buckling problem are Case 1: the crosssections are doubly symmetric about the $y$ and $z$ coordinate axes. Case 2: the cross-section is singly symmetric (monosymmetric) about one coordinate axis. 
4.2 Case 1: Buckling of columns with doubly symmetric cross-sections

Typical examples of doubly symmetric cross-sections are symmetric I sections and cruciform sections. For generalized column buckling problems involving doubly symmetric crosssections, $e_{y}=e_{z}=0$; since the shear centre is coincident with the centroid. The stability equation simplifies to:

$$
\left|\begin{array}{ccc}
\left(Q_{z z}-N_{x}\right) & 0 & 0 \\
0 & \left(Q_{y y}-N_{x}\right) & 0 \\
0 & 0 & \frac{I_{E}}{A}\left(Q_{\phi}-N_{x}\right)
\end{array}\right|=0
$$
(85):

$\left(Q_{z z}-N_{x}\right)\left(Q_{y y}-N_{x}\right)\left(Q_{\phi}-N_{x}\right) \frac{I_{E}}{A}=0$

The buckling modes are uncoupled (decoupled).

The three roots (zeros) of the characteristic buckling equation become:

$N_{x}=Q_{z z}=E I_{z z}\left(\frac{n \pi}{l}\right)^{2}$

$N_{x}=Q_{y y}=E I_{y y}\left(\frac{n \pi}{l}\right)^{2}$

$N_{x}=Q_{\phi}=\frac{A}{I_{E}}\left(E C_{w}\left(\frac{n \pi}{l}\right)^{2}+G J\right)$

\subsection{Case 2: Generalised elastic buckling of columns with monosymmetric cross-sections}

Typical example of column with monosymmetric crosssection is column with channel section $\sqcup$. If the $z z$ axis is the axis of symmetry, then $e_{y}=0, e_{z} \neq 0$ and the buckling equation becomes:

$$
\left|\begin{array}{ccc}
\left(Q_{z z}-N_{x}\right) & 0 & -N_{x} e_{z} \\
0 & \left(Q_{y y}-N_{x}\right) & 0 \\
-N_{x} e_{z} & 0 & \frac{I_{E}}{A}\left(Q_{\phi}-N_{x}\right)
\end{array}\right|=0
$$

The characteristic buckling equation becomes from Equation (84), putting $e_{y}=0$,

$\left(Q_{y y}-N_{x}\right)\left[\left(Q_{z z}-N_{x}\right)\left(Q_{\phi}-N_{x}\right)-N_{x}^{2} \frac{e_{z}^{2}}{r_{0}^{2}}\right]=0$

Solving, we have two possibilities for the roots of Equation (91):

$N_{x}=Q_{y y}=E I_{y y}\left(\frac{n \pi}{l}\right)^{2}$

or, $\left(Q_{z z}-N_{x}\right)\left(Q_{\phi}-N_{x}\right)-N_{x}^{2} \frac{e_{z}^{2}}{r_{0}^{2}}=0$

\section{DISCUSSION}

The least squares weighted residual method has been successfully used in this work to solve the generalised elastic column buckling problem for the case of pinned supports at the ends. The generalized elastic column buckling problem is represented as a system of three coupled ordinary non-linear differential equations with variable coefficients given by Equations (1-3) in the most general problem. The buckling problem considered in this study assumed prismatic crosssections, homogeneous materials and further assumed that the $y z$ coordinates are principal coordinates yielding $I_{z z}=0$. It also assumed that the column is free form transverse loads and torques yielding a simplification of the governing ordinary differential equations to a system of three coupled linear ODEs with constant parameters given by Equations (16-18). The governing equations solved by the least squares weighted residual method were presented as a set of three functionals to be minimized as Equations (24)-(26). The least squares weighed residual variational statements of the problem were given as the Equations (30)-(32). In terms of the shape functions, the least squares weighted residual variational statements were given in general as Equations (33)-(35).

For the elastic buckling problem where the ends $x=0$ and $x=l$ are on pinned supports, the three suitable displacement functions that satisfied all the geometric and force boundary conditions (Equations (36)-(47)) were given as Equations (45)-(50). The displacement shape function was thus given by Equation (51) for all possible buckling modes.

The substitution of the approximate displacement functions gave the least squares weighted residual variational integrals as the three equations, Equations (56-58). Further simplification of the least squares weighted residual variational integral statements reduced the BVP of generalized elastic column buckling to the algebraic eigenvalue eigenvector problem given by Equations (66)-(68); a system of homogeneous linear algebraic equations in terms of the modal displacement amplitudes. The homogeneous algebraic eigenvalue eigenvector problem was presented in matrix form as Equation (69).

The condition for non-trivial solution of homogeneous algebraic equations was applied to find the buckling (stability) equation as Equation (70). The characteristic buckling equation was further expressed in terms of the Euler buckling load expressions about the two axes ( $y y$ and $z z$ axes) of the cross-section and pure torsional buckling load $Q_{\phi}$ as Equation (75). Expansion of the stability equation and algebraic simplification yielded the characteristic buckling equation for the general asymmetric cross-section as the third order polynomial in terms of $N_{x}$ given by Equation (82). The characteristic buckling equation for asymmetric section was further simplified as Equation (83) or (84). The determination of the three roots of the characteristic buckling equation yield the three possible buckling loads, with the least buckling load governing the behaviour.

Two special cases were further considered for the generalised elastic column buckling problem involving doubly symmetric cross-sections and simply symmetric crosssections. The characteristic buckling equation for doubly symmetric cross-sections was obtained as Equation (86), a third order polynomial. Solution showed the roots and buckling modes are uncoupled, and the buckling could be by pure Euler flexural buckling about the $z z$ axis or about the $y y$ 
axis or by pure torsional buckling as given by Equations (87)(89).

The lowest value of the three buckling loads is the critical buckling load, and would determine how the elastic column with doubly symmetric cross-section would fail.

For the generalized elastic column problem involving singly symmetric cross-section, the case where the column is symmetrical about the $z z$ axis was considered. The characteristic buckling equation for this case was found as Equation (90). Expansion yielded the buckling equation as Equation (91), a third order polynomial equation in $N_{x}$ with one flexural buckling mode uncoupled and the other flexural buckling mode coupled with the torsional buckling mode. This shows that such columns with singly symmetric sections can fail by either Euler flexural buckling mode in the $y y$ direction (for the case of symmetry about the $z z$ axis), and/or flexural torsional buckling mode. The roots of the polynomial which are the eigenvalues give the values of the buckling loads. The three zeros of the polynomial would give three values of the buckling load. Euler flexural buckling load about the $y y$ axis given by Equation (92) and two coupled flexural - torsional buckling loads found by solving Equation (93). The expressions for the characteristic buckling loads obtained by the least squares weighted residual method agree excellently with solutions by Det [27], Wang et al. [26], Mama et al. [3] who used the finite Fourier sine transform method, Onah et al. [2] who used the Fourier series method and Ike et al. [4] who used the Galerkin variational method.

\section{CONCLUSION}

The conclusions of the study are as follows:

i. The least squares weighted residual method can efficiently solve the BVP presented by the system of linear ordinary differential equations subject to the boundary conditions for the generalized elastic column buckling problem for pinned ends at $x=0$, and $x=l$.

ii. The method simplified the problem from a BVP to an algebraic eigenvalue problem represented by a system of homogeneous algebraic equations.

iii. The characteristic buckling equation was found as a third order polynomial in terms of $N_{x}$; whose three roots gave the three buckling loads.

iv. In generalised elastic column buckling problems with doubly symmetric cross-sections, the resulting system of algebraic homogeneous equations are uncoupled yielding characteristic buckling equations with uncoupled buckling loads, and uncoupled buckling modes.

v. In generalised elastic column buckling problems with singly symmetric cross-sections, with $z z$ axis as the axis of symmetry, the flexural buckling mode about the $y y$ axis is uncoupled, while the flexural buckling mode about the $z$ axis, is coupled with torsional buckling.

vi. In generalised elastic column buckling problems with asymmetric cross-sections, the three buckling modes are coupled and the two flexural buckling modes about the $y y$ and $z z$ axes interact with the torsional buckling mode.

vii. The expressions obtained for the characteristic buckling equations were exact solutions since the exact displacement shape functions that satisfied all the geometric and force boundary conditions were used.

\section{REFERENCES}

[1] Torsion in Structural Design. http//people.virginia. edu/ttb/torsion.pdf. pp.73, accessed on 01/01/2017.

[2] Onah HN, Ike CC, Nwoji CU. (2017). Flexural torsional buckling analysis of thin walled columns using the Fourier series method. International Journal of Advanced Engineering Research and Science (IJAERS) 4(3): 292-298. https//dx.doi/10.22161/ijaers.4.3.45

[3] Mama BO, Ike CC, Nwoji CU, Onah HN. (2017). Application of the finite Fourier sine transform method for the flexural - torsional buckling analysis of thinwalled columns IOSR. Journal of Mechanical and Civil Engineering (IOSR-JMCE) 14(2): 51-60. https://doi.org/10.9790/1684-1402015160

[4] Ike CC, Nwoji CU, Ikwueze EU, Ofondu IO. (2017). Solution of the generalised elastic column buckling problem by the Galerkin variational method. International Journal for Research in Applied Science and Engineering Technology (IJRASET) 5(1): 468-475.

[5] Timoshenko SP, Gere JM. (1961). Theory of elastic stability. McGraw Hill Koga Kusha Ltd New York.

[6] Al-Sheikh AMS. (1985). Behaviour of thin-walled structures under combined loads. PhD Thesis Loughborough University of Technology.

[7] Riley CE. (2003). Elastic buckling loads of slender columns with variable cross-sections by the Newmark method. MSc Thesis, Department of Civil Engineering Colorado State University.

[8] Euler L. (1759). Sur la force des colonnes. Momoires de l'académie des sciences de Berlin 13: 252-282. in Opera Omnia set 2 17: 89-118.

[9] St Venant AJCB. (1855). Memoire sur la torsion des Prismes Mem Divers Savants 14: 233-560.

[10] Michell AGM. (1899). Elastic stability of long beams under transverse forces. Philos Mag 48 th Series 5 48(292): https://doi.org/10.1080/14786449908621336

[11] Prandtl L. (1899) Kipperscheinungen. Doctoral Dissertation des Universitat München.

[12] Timoshenko SP. (1905). On the stability in pure bending of a T beam. Bull Pol. Ins St Petersburg, pp. 4-5.

[13] Timoshenko SP. (1936). Theory of Elastic Stability. McGraw Hill, New York.

[14] Timoshenko SP. (1945). Theory of bending torsion and buckling of thin-walled members of open cross-section. Journal Franklin Institute 239(3): 201-219. https://doi.org/10.1016/0016-0032(45)90093-7

[15] Wagner H. (1936). Torsion and Buckling of open sections. Technical Memorandum No 807 US National Advisory Committee for Aeronautics, p. 18.

[16] Vlasov VZ. (1961). Thin walled elastic beams English translation. National Science Foundation, Washington DC London Oldbourne Press.

[17] Alsayed SH. (1987). Inelastic behaviour of single angle columns. PhD Thesis, The University of Arizona University Microfilms International http//hdl.handlev.net/10150/184041.

[18] Zlatko TZ. (2012). Stress and strain deflection of an open profile thin walled beam at constrained torsion by boundary element method. Journal of Theoretical and Applied Mechanics Sophia 42(2): 43-54. https://doi.org/10.2478/v10254-012-0007-y 
[19] Trahair NS. (1993). Flexural - Torsional Buckling of Structures. CRC Press Ann Arbor.

[20] Trahair NS. (2016). Torsion equations for lateral buckling Research report R 964 July 2016 School of Engineering, The University of Sydney.

[21] Allen HG, Bulson PS. (1980). Background to Buckling. McGraw Hill Book Company, London.

[22] Chajes A. (1974). Principles of structural stability theory. Prentice Hall New Jersey.

[23] Avcar M. (2014). Elastic buckling of steel columns under axial compression. American Journal of Civil $\begin{array}{lll}\text { Engineering } & \text { 2(3): }\end{array}$ https://doi.org/10.11648/j.ajce.20140203.17

[24] Nwakali JA. (1990). The collapse behaviour of double layer space trusses incorporating eccentrically loaded tee-section members. Ph.D Thesis Department of Civil Engineering University of Surrey, November 1990.

[25] Howlett JN. (1972). An investigation into the structural behaviour of thin walled aluminium alloy welded battered struts. MSc Thesis University of Durham July 1972 available at Durham E. Thesis online http//ethesis.dur.ac.uk/10293

[26] Wang CM, Wang CY, Reddy JN. (2005). Exact solution for buckling of structural members. CRC series in Computational Mechanics and Applied Mechanics CRC Press, USA.

[27] Det Norske Veritas (2004). Buckling strength analysis of bars and frames, and spherical shells. Classification Notes 30: 15.

[28] Zhu S. (2009). Elastic flexural torsional buckling analysis of doubly symmetrical web tapered beams. MSc Thesis University of Pittsburg Oct. 2009, p. 208.

\section{NOMENCLATURE}

$x$

$l$

$y z$

A

$t(x)$

$M_{l z} M_{l y}$

$J$

$E$

G

$I_{y y} I_{z z}$

$C_{w}$ longitudinal (axial) coordinate variable length of the column plane of the cross-section of the column area of the cross-section

applied torque

moments due to transverse loads

Saint Venant torsional stiffness of the crosssection

Young's modulus of elasticity of the column material

shear modulus or modulus of rigidity

moments of inertia about the $y y$ and $z z$ coordinate axes

warping constant $n$

$I_{E}$

$I_{y z}$

$e_{y}, e_{z}$

$V_{y}, V_{z}$

$q_{y}, q_{z}$

$N_{x}$

$v(x) w(x)$

$\theta(x)$

$H_{y}, H_{z}, H_{w}, C_{y}, C_{z}$, geometrical properties of the crosssection

$F_{1}, F_{2}, F_{3} \quad$ functionals to be minimized

$\bar{v}(x), \bar{w}(x), \bar{\theta}(x)$ approximations to the unknown displacement functions

$\varphi_{n}(x) \quad$ displacement functions for the $n$th buckling

$Q_{z z} \quad$ mode $\quad$ Euler flexural buckling load about the $z z$ axis

$Q_{y y} \quad$ Euler flexural buckling load about the $y y$

axis

$Q_{\phi} \quad$ torsional buckling load

$r_{0} \quad$ radius of gyration

$A_{n}, B_{n}, \theta_{n} \quad$ buckling modal amplitudes of the unknown displacement $v(x), w(x)$ and $\theta(x)$ for the $n$th mode

$\frac{\frac{d}{d x}}{\frac{d^{n}}{d x^{n}}}$

$L_{1}=\frac{d^{4}}{d x^{4}}$

$L_{2}=\frac{d^{2}}{d x^{2}}$

$\iint_{A}$

$\int_{0}^{l}$

$\sum_{n=1}^{\infty}$

$v^{\prime \prime}(x)$

$w^{\prime \prime}(x)$

$\theta^{\prime \prime}(x)$ first ordinary differential operator

$n$th ordinary differential operator

notation for the fourth ordinary differential operator

notation for the second ordinary differential operator

double integral over the domain of the cross

sectional area, $A$

intergral over the length $l$ of the column

summation for $n=1,2,3, \ldots, \infty$

second derivative of $v(x)$ with respect to $x$ second derivative of $w(x)$ with respect to $x$ second derivative of $\theta(x)$ with respect to $x$ 